\title{
Detecting Thoracic Diseases via Representation Learning with Adaptive Sampling ${ }^{\text {th }}$
}

\author{
Hao Wang ${ }^{\mathrm{a}, 1}$, Yuan-Yuan Yang ${ }^{\mathrm{b}, 1}$, Yang Pan ${ }^{\mathrm{c}}$, Peng Han ${ }^{\mathrm{d}}$, Zhong-Xiao Li ${ }^{\mathrm{d}}$, He-Guang Huang ${ }^{\mathrm{b}, 2}$, Shun-Zhi Zhu ${ }^{\mathrm{e}, 2}$ \\ ${ }^{a}$ Inception Institute of Artificial Intelligence, UAE \\ ${ }^{b}$ Department of General Surgery, Fujian Medical University Union Hospital, China \\ ${ }^{c}$ Department of Geriatrics, Nanjing Brain Hospital, China \\ ${ }^{d}$ Computer, Electrical and Mathematical Science and Engineering Division, KAUST, Saudi Arabia \\ ${ }^{e}$ School of Computer and Information Engineering, Xiamen University of Technology, China
}

\begin{abstract}
The recently released chest X-ray dataset, ChestX-ray14, has attracted more and more attention on automatic detection of thoracic diseases. In this work, we use deep learning techniques to develop a multi-class classifier. Given a chest X-ray image as input, the classifier outputs a vector of probability values, of which each component corresponds to the probability of having one specific thoracic disease. The merit of our proposed solution is based on several major observations of the ChestX-ray 14 data. First, the diversity in ChestX-ray14 is much smaller than that in other natural image datasets such as ImageNet due to very similar global outlines of chest X-ray images. Second, ChestX-ray14 is much more imbalanced than the datasets considered in most existing studies. The size of the largest class is 87.57 times larger than that of the smallest class. Third, from the application perspective, the task is not really cost-sensitive to misclassifications, thus it is difficult to manually fix weights for different misclassifications. To deal with these difficulties, we propose an adaptive sampling method that monitors the performance of the model during training and automatically increase the weight of relatively poorly performed classes. Extensive experiments demonstrate that our proposed method outperforms the state-of-the-art algorithms.
\end{abstract}

Keywords: Thoracic disease detection, Deep learning, Imbalanced learning, Adaptive sampling

\section{Introduction}

Thoracic diseases refer to a large collection of diseases $10-{ }^{22}$ cated in the thoracic cavity. Despite of various rare cases, ${ }^{23}$ certain thoracic diseases, such as pneumonia, pneumothorax, ${ }^{24}$ edema, etc., are very common in clinical practice. For exam- ${ }^{25}$ ple, it has been reported that pneumonia is the single largest ${ }^{26}$ cause of death in children worldwide, accounting for approx- ${ }^{27}$ imately $20 \%$ of childhood deaths [43]. Chest X-ray is one of ${ }^{28}$ the most commonly used imaging tools to detect thoracic diseases in clinical practice due to its wide availability and cost- ${ }^{30}$ effectiveness. Still, it requires sufficient knowledge and experi- ${ }^{31}$ ence to accurately interpret an X-ray image. Indeed, the process ${ }^{32}$ of $X$-ray interpretation involves complex decision-making with ${ }^{33}$ various uncertainties, thus even the most experienced physi- ${ }^{34}$ cians/radiologists may have disagreement in the interpretation ${ }^{35}$ of the same X-ray image [3, 9].

Since the number of experienced professionals is always ${ }^{37}$ limited, artificial intelligence (AI) techniques become practi- ${ }^{38}$ cally important, attracting lots of attention [8, 36, 45]. For ex- ${ }^{39}$ ample, unsupervised (spectral) learning has been widely used in ${ }^{40}$

\footnotetext{
This work is supported by Joint Funds of Scientific and Technological In- ${ }^{42}$ novation Program of Fujian Province (No. 2017Y9059), Sail Fund of Fujian 43 Medical University (No. 2017XQ1027), "Ethicon Excellence in Surgery” Grant 44 of Wujieping Medical Foundation (No. 320.2710.1801), and the Science and Technology Planning Project of Xiamen/Quanzhou City (No. 3502Z20183055, 2017G030).

${ }^{1}$ Hao Wang and Yuan-Yuan Yang are the co-first authors.

${ }^{2}$ He-Guang Huang and Sun-Zhi Zhu are the corresponding authors.
}

various image processing tasks [29, 48, 50, 51]. Recently, many deep learning techniques have been developed [31] and successfully applied in various medical image analysis tasks [22]. Such success of deep learning mainly comes from the advances of big data techniques, which reduce the cost of collecting and storing huge amount of training data, as well as the advances of computer hardware such as GPUs, which enhance the machines with greater computing power.

With data and computing power, deep learning offers not only an end-to-end solution, directly from image inputs to diagnoses, but also a systematic way to learn the representations of data. Indeed, representation learning (a.k.a. feature learning) is important because the success of machine learning largely depends on data representations [2, 49]. Conventionally, data representations of medical images are designed via interdisciplinary collaborations between medical professionals and AI researchers. Such handcrafted data representations certainly capture some important features for accurate diagnoses (see, e.g., [27]); nonetheless, due to the uncertain nature of the clinical interpretation process, it remains open whether the representations are good enough for machine learning approaches. Considering this, representation learning provides an alternative to automatically discover a good representation of data, which may lead to an expert-level accuracy in diagnoses [22].

In this paper, we aim at accurate detection of common thoracic diseases via representation learning using the recent ChestX-ray14 dataset [41], which is a hospital-level dataset 
containing 112,120 frontal-view chest X-ray images of 30,805103 unique patients. There are 14 common pathological keywords 104 (i.e., labels) involved in the dataset, and each image is attached ${ }_{105}$ with either some (one or more) of the 14 labels or a single la-106 bel of 'No Finding'. Since the release of ChestX-ray14, several 107 deep learning solutions have been proposed to detect thoracic ${ }_{108}$ diseases. Wang et al. [41] built a unified framework upon sev-109 eral deep models pre-trained with ImageNet [6]. Yao et al. [44] $]_{110}$ proposed a multi-class classifier, based on the long short-term 11 memory (LSTM) architecture [13], considering the interrela-112 tionships between the 14 labels. Rajpurkar et al. [32] proposed ${ }_{113}$ CheXNet, which is essentially a 121-layer DenseNet [14]. Mao114 et al. [23] built a deep generative model based on several pre- -115 trained encoders. Wang et al. [42] developed a text-image em-116 bedding network, TieNet, based on an argument that not only ${ }_{117}$ the X-ray images but also the text reports are useful.

To the best of our knowledge, all the state-of-the-art solu-119 tions overlook the fact that the ChestX-rayl4 dataset is highly $y_{120}$ imbalanced. For example, there are 19,871 (17.72\%) samples 121 labeled with 'Infiltration' but merely $227(0.20 \%)$ labeled with ${ }_{122}$ 'Hernia'. It is well known that such a skewed class distribution may have a major impact on the practical performance of classifiers [11]. Existing solutions to imbalanced learning mainly in- ${ }^{123}$ volves data sampling techniques and cost-sensitive approaches. ${ }_{124}$ For example, Jeatrakul et al. [17] used a complementary neural ${ }_{125}$ network, consisting of a pair of feed-forward back-propagation ${ }_{126}$ neural networks, to implement under-sampling; Khan et al. [20] $]_{127}$ introduced cost-sensitive considerations into the loss functions, ${ }_{128}$ where the class-sensitive costs are learned from data during the ${ }_{129}$ training process; Ren et al. [33] proposed a meta-learning approach to reweight the training examples. Unfortunately, all ${ }_{130}$ of these solutions were designed to perform general-purpose classifications and none of them is tailored for the detection of ${ }^{131}$ thoracic diseases with the ChestX-ray14 dataset. Our task chal- ${ }^{132}$ lenges the existing imbalanced learning solutions since:

- The diversity in ChestX-ray14 is much smaller than that ${ }^{135}$ in other natural image datasets such as ImageNet, a basic ${ }^{136}$ data resource of many deep learning solutions. This is ${ }^{137}$ because the frontal-view chest X-ray images tend to have ${ }^{138}$ highly similar global outlines, only with small (yet very ${ }^{139}$ important) differences in local areas.

- ChestX-ray 14 is much more imbalanced than the datasets ${ }^{14}$ considered in most existing studies (e.g., those in [20]). ${ }^{142}$ The imbalance ration of ChestX-ray 14 generally leads to very large weights on minority classes since the weights ${ }^{143}$ should, in principle, be inversely proportional to the sizes ${ }^{144}$ of classes. It is well known that large weights tend to ${ }^{145}$ make the learning process unstable, even divergent [16]. ${ }^{146}$

- From the application perspective, the task is not really ${ }^{147}$ cost-sensitive to misclassifications. In fact, any misclas- ${ }^{148}$ sification of the thoracic diseases should be considered ${ }_{150}^{149}$ equally unfortunate. Thus, hardly any prior knowledge ${ }_{151}$ is available if one wants to modify the loss functions or ${ }^{151}$ design the reweighting schemes. via adaptive sampling. The basic idea is that we dynamically change the weights of different classes in the training process. Specifically, we monitor the performance of the model during each training iterations. Whenever the model is has a relatively poor performance on a class, that class will be emphasized more during the next training iteration. Our main contributions are the following. First, we build a DenseNet-based deep structure as the model for multi-class classification. Second, we propose an adaptive data augmentation technique to handle the data imbalance problem. Last but not least, we experimentally compare our proposed method with the state-of-the-art solutions on the ChestX-ray 14 dataset. The results confirm the advantage of our proposed method.

The rest of this paper is organized as follows. Section 2 reviews some studies related to our research. Section 3 shows the basic statistics, especially the high skewness, of ChestXray 14 and presents a formal problem formulation. Section 4 explains our proposed method in detail. Section 5 compares our proposed method with the existing solutions. Finally, Section 6 concludes the paper.

\section{Related Work}

Computer-aided diagnosis (a.k.a computer-aided detection, CAD) has long been an active research and application direction, of which the most recent advances are usually recognized as medical AI solutions. In addition to those studies mentioned in Section 1, the following lines of research are related to our work in general.

\subsection{Signal Processing for $C A D$}

Parveen and Sathik [30] employed wavelet and Fourier transform techniques to extract features from chest X-ray images, based on which a fuzzy $c$-means clustering was used to detect pneumonia. Ellington et al. [7] carried out a computerized lung sound analysis for paediatric pneumonia diagnosis. Morillo et al. [26] used short-term Fourier transform analysis to extract features from the recorded respiratory sounds. Then, after the dimesionality reduction using principal component analysis (PCA), they trained a probabilistic neural network (PNN) to detect pneumonia in patients with chronic obstructive pulmonary disease (COPD). These line of work typically relies on signal-level features, thus the focus is very different from ours.

\subsection{Conventional Machine Learning for CAD}

During the past decades, there have been several comparative studies of conventional machine learning methods. Cooper et al. [5] compared eight statistical and machine learning methods, including neural networks, Bayesian classification, etc., on their performance in predicting mortality of patients with pneumonia. Chapman et al. [4] compared the performance of expert-crafted rules, a Bayesian network, and a decision tree in detecting bacterial pneumonia based on the chest X-ray reports. Sousa et al. [39] showed that support vector machines (SVMs) outperformed naïve Bayes and $k$-nearest neighbor classifiers in

In this work, we tackle the imbalanced learning problem 
the detection of childhood pneumonia using chest X-ray images. Naydenova et al. [27, 28] considered applying different feature selection techniques, including LASSO [34], sparse linear discriminant analysis (sLDA) [25], etc., on dozens of clinical characteristics. The selected features were then used to train machine learning models, such as SVMs and random forests, to detect childhood pneumonia. In addition, Jun et al. [18] trained an SVM to detect lung diseases based on the texture and shape features of high-resolution computed tomography (CT) images.

\subsection{Deep Learning for $C A D$}

In recent years, deep learning has been widely adopted in CAD. Most of them also adopt transfer learning techniques to handle the data sparsity problem. Ausawalaithong et al. [1] employed a 121-layer DenseNet for lung cancer prediction, where they considered transfer learning between the ChestX-ray14, JSRT [37] and ImageNet datasets. Kermany et al. [19] transferred a convolutional neural network (CNN) model pre-trained from ImageNet for the screening of patients with common treat ${ }^{205}$ able blinding retinal diseases. Then, they showed the generality of their method by transferring the model for the detection of ${ }^{207}$ pediatric pneumonia using chest X-ray images. Haloi, Rajalak- ${ }^{208}$ shmi, and Walia [10] proposed a confidence-aware deep learning system to distinguish between pneumonia and tuberculosis. ${ }^{211}$ Transfer learning techniques were used to make full use of sev- ${ }^{21}$ eral different datasets. Zech et al. [46] considered the trans ${ }^{2}{ }^{212}$ fer performance of the $\mathrm{CNN}$ models between X-ray images ob- ${ }^{213}$ tained from different hospitals. They found that the CNN models tended to achieve better internal than external performance.

Recently, Saraiva et al. [35] used a two-phase method to imple-215 ment pneumonia category classification. First, a fully convolutional network (FCN) model is used to segment the images into ${ }^{216}$ regions. Then, a deep CNN model is used to classify the target 217 lung regions. It is argued that the automatically extracted fea-218 tures outperform the manually chosen ones. For state-of-the-art219 advances of deep learning for medical image analysis, inter-220 ested readers are further referred to recent surveys by Litjens et221 al. [22] and Mazurowski et al. [24].

\section{Data and Problem Formulation}

The ChestX-ray14 dataset [41] contains 112,120 frontal-226 $^{226}$ view chest X-ray images of 30,805 unique patients aged from ${ }^{227}$ 1 to $95^{3}$, in which $63,340(56.49 \%)$ are males and $48,780^{228}$ $(43.51 \%)$ are females. There are in total 15 different labels in $^{229}$ the dataset (14 common pathological keywords plus 'No Find-230 ing'). Figure 1 shows the distribution of classes in the ChestX-231 ray14 dataset. Note that the percentage values do not sum up ${ }^{232}$ to 1 because one image may be labeled with multiple classes. ${ }^{233}$ As can be seen, the ChestX-ray14 dataset is very imbalanced. ${ }^{234}$ Over $53.83 \%$ of the images are without any class label (i.e., la ${ }^{235}$ beled 'No Finding'). Major classes such as 'Infiltration' have ${ }^{236}$ sizes up to 87.54 times larger than that of minor classes such $^{237}$ as 'Hernia'. In fact, the average and standard deviation of the ${ }^{238}$

\footnotetext{
${ }^{3} 16$ age values are found abnormal $(\geq 148)$ and are thus ignored.
}

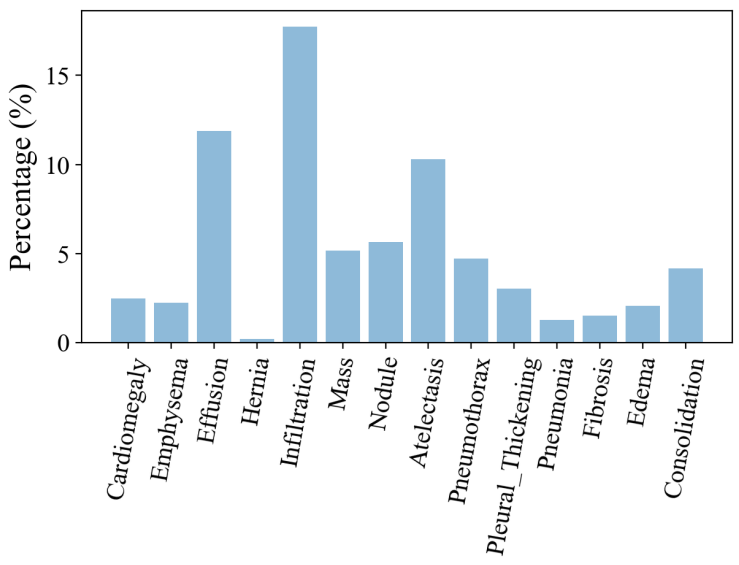

Figure 1: The imbalanced class distribution in ChestX-ray14.

percentage values of different class labels are $8.42 \%$ and 0.134 , respectively.

Problem formulation. Given ChestX-ray14 as the training dataset, our task is to train a classifier $\mathcal{M}$, such that for each input image $\boldsymbol{X}, \mathcal{M}(\boldsymbol{X})$ returns a 14-dimensional vector, $\boldsymbol{p}^{\top}$, each element of which represents the probability of $\boldsymbol{X}$ being labeled with the corresponding disease. Note that if each component $p_{k} \approx 0$, the image $\boldsymbol{X}$ belongs to the 15 th class of 'No Finding'. We aim at developing a good classifier $\boldsymbol{C}$ in the sense that it can produce more accurate predictions.

\section{Our Method}

\subsection{Network structure}

As is mentioned in Section 1, the diversity of the input Xray images are small since they tend to have similar global outlines with small local differences. Thus, to capture the details in the X-ray images, we employ multi-layer networks. Specifically, we use DenseNet-121, in which the input features, including the original images, have more connections with higher layers and objective functions, which could make the identification of tiny pathology possible. The architecture of DenseNet-121 is illustrated in Figure 2.

As we can see, the main structure of the network is 4 consecutive dense blocks connected by 3 transition layers.

$\boldsymbol{\ell}$-layer dense blocks. An $\ell$-layer dense block consists of $\ell$ dense layers. The $j$-th dense layer can be seen as a nonlinear function $\boldsymbol{H}_{j}(j=1,2, \cdots, \ell)$, converting the input features $\boldsymbol{x}_{0}, \boldsymbol{x}_{1}, \cdots, \boldsymbol{x}_{j-1}$ into an output feature $\boldsymbol{x}_{j}$. Specifically, the function $\boldsymbol{H}_{j}$ is implemented as the consecutive execution of the sequence BN-ReLU-Conv $(1 \times 1)-B N-R e L U-C o n v(3 \times 3)$. Here, BN stands for batch normalization [16]. There are 4 dense blocks with $6,12,24,16$ layers, respectively, used in the network architecture.

Transition layers. The 4 dense blocks are connected by 3 transition layers. Each transition layer consists of a BN-ReLU$\operatorname{Conv}(1 \times 1)$ sequence followed by an average pooling layer. 


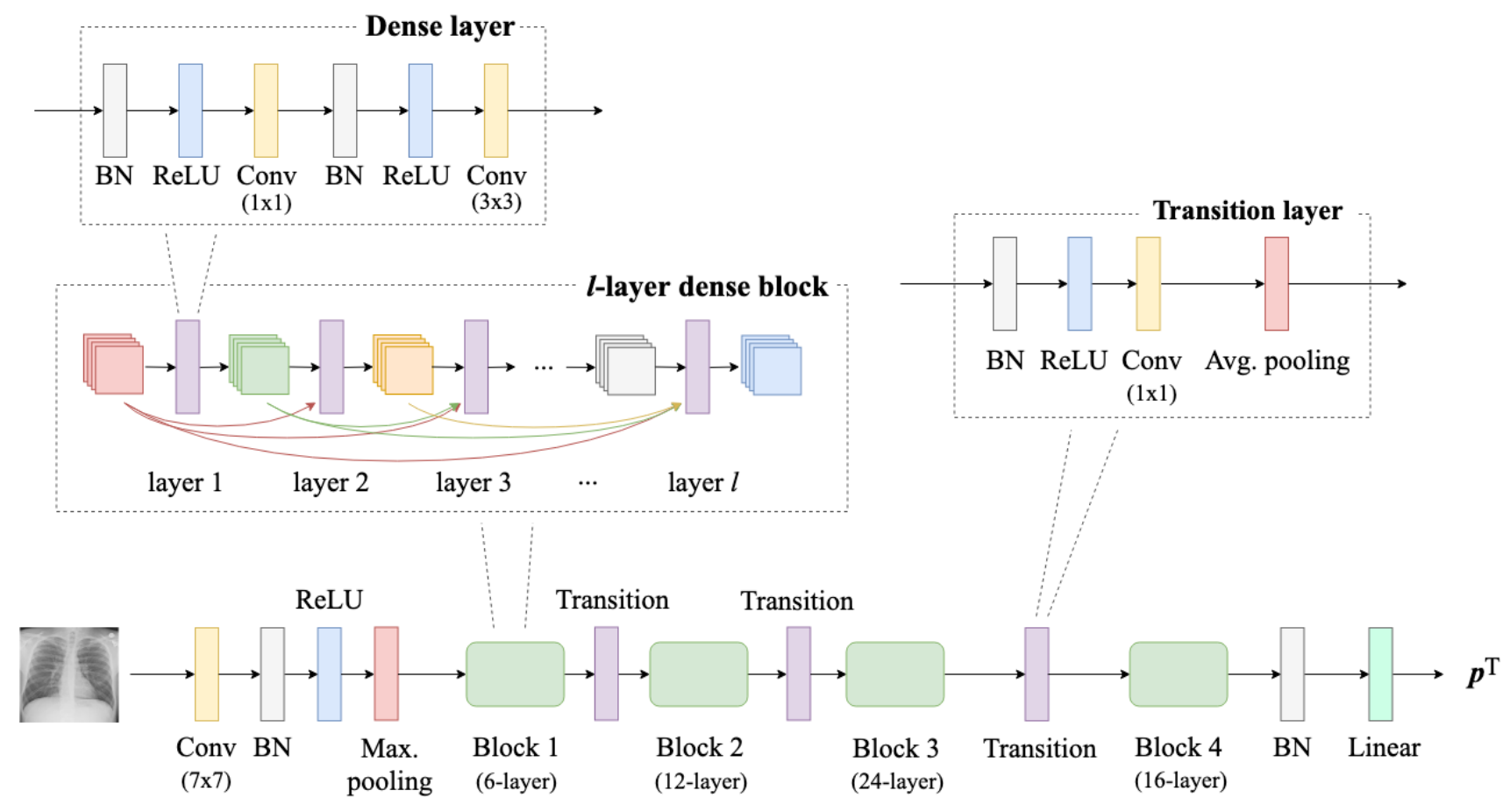

Figure 2: Deep network architecture.

First layers. Before the first dense block, the very first layers of the network are a sequence of $\operatorname{Conv}(7 \times 7)$-BN-ReLu followed by a max pooling layer.

Last layers. After the last dense block, a BN layer and a linear layer are used to produce the final scores (i.e., probabilities) $\boldsymbol{p}^{\top}$.

\subsection{The Training Process}

Given the deep network model $\mathcal{M}$ as shown in Figure 2, the training process is presented as Algorithm 1. As can be seen, Algorithm 1 takes the inputs of the deep model $\mathcal{M}$, the training dataset $D^{\mathrm{tr}}=\bigcup_{k=1}^{K} D_{k}^{\mathrm{tr}}$, and the validation dataset $D^{\mathrm{val}}=\bigcup_{k=1}^{K} D_{k}^{\mathrm{val}}$, where $K$ is the total number of classes. $D_{k}^{\mathrm{tr}}$ and $D_{k}^{\text {val }}$ are the training and validation dataset of the $k$-th class, respectively. Each data sample is of the form $\left(\boldsymbol{X}, \boldsymbol{y}^{\boldsymbol{\top}}\right)$, where $\boldsymbol{X}$ is the image and $\boldsymbol{y}^{\boldsymbol{\top}} \in\{0,1\}^{K}$ indicates the set of labels. The $k$-th component of $\boldsymbol{y}^{\top}, y_{k}$, takes value 1 if $\boldsymbol{X}$ belongs to the $k$-th class and 0 otherwise. Note that $D_{i}^{\mathrm{tr}} \cap D_{j}^{\mathrm{tr}} \neq \emptyset$ is possible even if $i \neq j$ since a data item $\boldsymbol{x}$ may have multiple class labels.

The training process consists of a total number of $T$ iterations. Our key idea of handling data imbalance is to bias towards those classes with relatively poor performance. To that end, we propose an adaptive data augmentation (i.e., oversampling) technique. Specifically, for the $k$-th class we set up an augmentation ratio $\delta_{k} \geq 1$, which is initially 1 (Line 1), to control how the training data of the $k$-th class, $D_{k}^{\text {tr }}$, is augmented. In each iteration, we first augment $D_{k}^{\text {tr }}$ to make it $\delta_{k}$ times larger by randomly duplicating its data samples (Lines 3-4). Then, we shuffles the augmented dataset $D^{\mathrm{tr}}=\bigcup_{k=1}^{K} D_{k}^{\mathrm{tr}}($ Line 5) and split $D^{\text {tr }}$ into equally-sized batches to facilitate model training

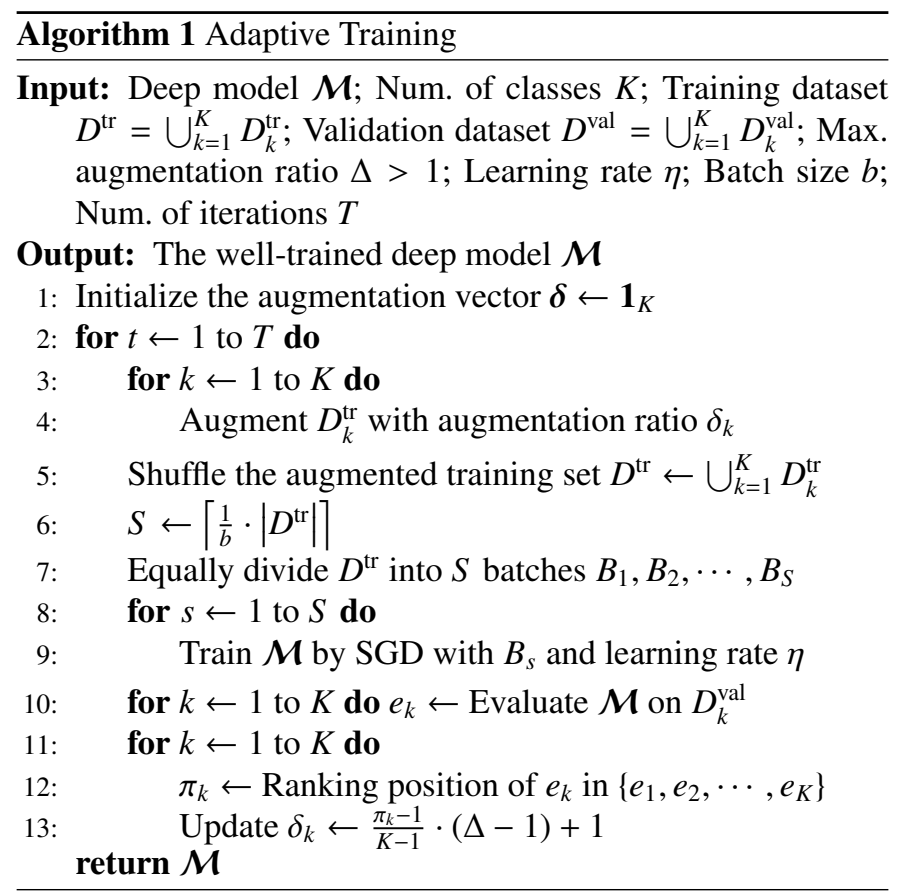

(Lines 6-7). Each batch is then fed into the model $\mathcal{M}$ where a stochastic gradient descent (SGD) algorithm is employed to tune the model $\mathcal{M}$ (Line 9). SGD is a standard and widelyused technique to solve optimization problems [47]. During the SGD process, the loss of generating a probabilistic prediction 
$\boldsymbol{p}^{\top}$ for a single training sample $\left(\boldsymbol{X}, \boldsymbol{y}^{\top}\right)$ is

$$
L\left(\boldsymbol{X}, \boldsymbol{y}^{\boldsymbol{\top}} ; \boldsymbol{p}^{\boldsymbol{\top}}\right)=\sum_{k=1}^{K}\left(-y_{k} \log p_{k}-\left(1-y_{k}\right) \log \left(1-p_{k}\right)\right),
$$

which is known as the unweighted binary cross entropy loss.285 Note that we use an unweighted loss since any misclassifica-286 tion is equally unfortunate in our application. The tuned model 287 $\mathcal{M}$ is then validated using the validation dataset $D_{k}^{\mathrm{val}}$. The performance of $\mathcal{M}$ on the $k$-th validation dataset, $e_{k}$, can thus be ${ }^{288}$ evaluated (Line 10). After that, we set $\pi_{k}$, taking values from ${ }^{289}$ $1,2, \cdots, K$, to be the ranking position of $e_{k}$ in the descending order of $\left\{e_{1}, e_{2}, \cdots, e_{K}\right\}$ (Line 12). For example, if $e_{1}=0.86,{ }^{29}$ $e_{2}=0.62$ and $e_{3}=0.9$, then $\pi_{1}=2, \pi_{2}=3$ and $\pi_{3}=1^{292}$ since $e_{3}>e_{1}>e_{2}$. If the $k$-th class has a large $\pi_{k}$ value, it ${ }^{293}$ means that the performance on the $k$-th class is relatively unsat ${ }^{294}$ isfactory. Therefore intuitively, we want to further focus on the ${ }^{295}$ training on the $k$-th class. This is implemented by updating the ${ }^{296}$ augmentation ratio $\delta_{k}$ by a factor of $\pi_{k}$ (Line 13). In particular, ${ }^{297}$ we update $\delta_{k}$ as

$$
\delta_{k}=\frac{\pi_{k}-1}{K-1} \cdot(\Delta-1)+1,
$$

To evaluate our proposed method, we conduct a set of ex-303 periments on the ChestX-ray14 dataset. We implemented our 304 method using PyTorch. Table 1 shows the parameters used in our implementation. Basically, for each parameter used in Algorithm 1 (i.e., the learning rate $(\eta)$, the maximum augmentation ratio $\Delta$, the batch size $b$, and the total number of iterations $T$ ), we first determine a wide range of candidates and then choose the best parameter setting that achieves the best practical performance.

Table 1: Parameters

\begin{tabular}{|r|l|}
\hline \multicolumn{1}{|c|}{ Parameters } & \multicolumn{1}{|c|}{ Range of values } \\
\hline Learning rate $\eta$ & $0.1,0.01,0.001$ \\
Max. augmentation ratio $\Delta$ & $2,4,8,16$ \\
Batch size $b$ & $32,64,128,256$ \\
Total num. of iterations $T$ & $5,10,15,20,25,30$ \\
\hline
\end{tabular}

We compared our method with the following three competitors:

1. SVM. This is the conventional SVM classifier using the histogram of oriented gradients (HOG) features. HOG counts the occurrences of gradient orientation in localized areas of an image. HOG features have been widely ${ }^{305}$ and successfully used in many object detection tasks.
2. LSTM. This method is proposed by Yao et al. [44] which uses an LSTM architecture as the basic classification model.

3. DCNN. This method is proposed by Wang et al. [41]. This method uses a unified deep convolutional neural network (DCNN) framework which includes ImageNet pretrained AlexNet [21], GoogleNet [40], VGGNet [38] and ResNet [12] as components.

We used exactly the same experiment setting with those of [44] and [41]. Note that comparing to DCNN, our model require no pre-training.

For each of the $K=14$ disease classes, we use the the Area Under the Curve (AUC) value to measure the performance, which is a widely used performance measure for imbalanced learning [15]. Specifically, AUC measures the area under the receiver operating characteristic (ROC) curve, of which the $\mathrm{x}$ axis and the $y$-axis are the false positive rate and the true positive rate, respectively. The ROC curve depicts relative tradeoffs between true positives and false positives. An AUC value is within the range of 0.5 to 1 , which can be interpreted as the probability of a randomly chosen positive instance being scored higher by the model than a randomly chosen negative instance. Hence, a higher AUC value indicates a better classifier.

Mathematically, given a classifier $\mathcal{M}$ and a test dataset $D^{\text {test }}$, for each $\boldsymbol{X}_{i} \in D^{\text {test }}, \boldsymbol{p}_{i}^{\top}=\boldsymbol{\mathcal { M }}\left(\boldsymbol{X}_{i}\right)$ is the $K$-dimensional vector of probabilistic prediction. Then, AUC of the $k$-th class is calculated as

$$
\operatorname{AUC}(\mathcal{M}, k)=\frac{\sum_{i=1}^{N_{k}^{+}} \sum_{j=1}^{N_{k}^{-}} \mathbb{I}\left(p_{i, k}>p_{j, k}\right)}{N_{k}^{+} N_{k}^{-}},
$$

where $N_{k}^{+}$and $N_{k}^{-}$are the number of positive and negative samples in the $k$-th class and $\mathbb{I}(\cdot)$ is the indicator function.

Table 2: AUC Values

\begin{tabular}{|r|l|l|l|l|}
\hline Pathology & SVM & LSTM & DCNN & Ours \\
\hline Atelectasis & 0.523 & 0.772 & 0.716 & $\mathbf{0 . 8 1 4}$ \\
Cardiomegaly & 0.514 & $\mathbf{0 . 9 0 4}$ & 0.807 & 0.899 \\
Effusion & 0.537 & 0.859 & 0.784 & $\mathbf{0 . 8 7 3}$ \\
Infiltration & 0.535 & 0.695 & 0.609 & $\mathbf{0 . 7 0 1}$ \\
Mass & 0.517 & 0.792 & 0.706 & $\mathbf{0 . 8 4 0}$ \\
Nodule & 0.513 & 0.717 & 0.671 & $\mathbf{0 . 7 7 5}$ \\
Pneumonia & 0.506 & $\mathbf{0 . 7 1 3}$ & 0.633 & 0.662 \\
Pneumothorax & 0.519 & 0.841 & 0.806 & $\mathbf{0 . 8 6 5}$ \\
Consolidation & 0.540 & 0.788 & 0.708 & $\mathbf{0 . 7 8 9}$ \\
Edema & 0.575 & $\mathbf{0 . 8 8 2}$ & 0.835 & 0.874 \\
Emphysema & 0.526 & 0.829 & 0.815 & $\mathbf{0 . 9 2 4}$ \\
Fibrosis & 0.513 & 0.767 & 0.769 & $\mathbf{0 . 8 0 9}$ \\
PT & 0.520 & 0.765 & 0.708 & $\mathbf{0 . 7 7 2}$ \\
Hernia & 0.595 & 0.914 & 0.767 & $\mathbf{0 . 9 2 3}$ \\
\hline Average & 0.53 & 0.80 & 0.74 & $\mathbf{0 . 8 2}$ \\
Std. Dev. & 0.025 & 0.071 & 0.070 & 0.079 \\
\hline
\end{tabular}

Table 2 shows the AUC values of SVM, LSTM, DCNN, and our proposed DenseNet-based adaptive sampling method, 
in which the performance values of LSTM and DCNN are re-367 ported in [32]. As can be seen, our proposed method achieves ${ }^{368}$ the best AUC performance in 11 out of the 14 cases. Even in $_{370}^{369}$ the rest 3 cases of cardiomegaly, pneumonia, and edema, the ${ }_{371}$ performance of our method is very close to the best perfor-372 mance. The results demonstrate the effectiveness of our pro- ${ }^{373}$ posed method.

\section{Conclusion}

A recently released chest X-ray dataset, ChestX-ray14, has ${ }_{380}$ attracted more and more attention on automatic detection of 381 thoracic diseases. In this work, we aimed at building an ef- ${ }^{382}$ fective multi-class classifier for automatic diagnosis of thoracic ${ }_{384}^{383}$ diseases. Our design was based on important observations of ${ }_{385}$ the ChestX-ray14 data. First, the diversity in ChestX-ray 14 is $^{386}$ very small. Second, ChestX-ray14 is very imbalanced. Third, ${ }_{388}^{387}$ from the application perspective, the task is not really cost- ${ }_{389}$ sensitive to misclassifications. Based on such observations, wез90 proposed an adaptive sampling method that can monitor the ${ }^{391}$ performance of the model during the training process and $\mathrm{au}_{-}{ }^{392}$ tomatically increase the weight of relatively poorly performed $\mathrm{d}_{394}$ classes. Extensive experiments showed that our method mays95 outperform the state-out-the-art solutions by a large margin.

\section{References}

[1] W. Ausawalaithong, A. Thirach, S. Marukatat, and T. Wilaiprasitporn. ${ }^{40}$ Automatic lung cancer prediction from chest $\mathrm{x}$-ray images using deep ${ }_{402}^{402}$ learning approach. arXiv: 1808.10858, 2018.

[2] Y. Bengio, A. Courville, and P. Vincent. Representation learning: a review ${ }^{404}$ and new perspective. TPAMI, 35(8):1798-1828, 2013.

[3] A. Brady, R. O. Laoide, P. McCarthy, and R. McDermott. Discrepancy ${ }^{406}$ and error in radiology: concepts, causes and consequences. Ulser Medicat ${ }^{407}$ Journal, 81(1):3-9, 2012.

[4] W. W. Chapman, M. Fizman, B. E. Chapman, and P. J. Haug. A compar- ${ }^{409}$ ison of classification algorithms to automatically identify chest X-ray re- ${ }^{410}$ ports that support pneumonia. Journal of Biomedical Information, 34:4- ${ }_{411}^{411}$ 14,2001

[5] G. F. Cooper, C. F. Aliferis, R. Ambrosino, J. Aronis, B. G. Buchanan, ${ }^{413}$ R. Caruana, M. J. Fine, C. Glymour, G. Gordon, B. H. Hanusa, J. E. ${ }^{414}$ Janosky, C. Meek, T. Mitchell, T. Richardson, and P. Spirtes. An eval- ${ }^{415}$ uation of machine-learning methods for predicting pneumonia mortality ${ }_{417}^{416}$ Artificial Intelligence in Medicine, 9(2):107-138, 1997.

[6] J. Deng, W. Dong, R. Socher, L.-J. Li, K. Li, and F.-F. Li. ImageNet: $a^{418}$ large-scale hierarchical image database. In CVPR, 2009.

[7] L. E. Ellington, R. H. Gilman, J. M. Tielsch, M. Steinhoff, D. Figueroa, ${ }^{420}$ S. Rodriguez, B. Caffo, B. Tracey, M. Elhilali, J. West, and W. Checkley. ${ }^{421}$ Computerised lung sound analysis to improve the specificity of paediatric ${ }^{422}$ pneumonia diagnosis in resource-poor settings: protocol and methods for ${ }^{423}$ an observational study. BMJ Open, 2(1):e000506, 2012.

[8] B. Erickson, P. Korfiatis, Z. Akkus, and T. L. Kline. Machine learning for ${ }^{425}$ medical imaging. Radiographics, 37(2):505-515, 2017.

[9] R. Fitzgerald. Error in radiology. Clinical Radiology, 56(12):938-946, ${ }^{427}$ 2001.

[10] M. Haloi, K. R. Rajalakshmi, and P. Walia. Towards radiologist-level ac- ${ }^{429}$ curate deep learning system for pulmonary screening. arXiv: $1807.031200^{430}$ 2018.

[11] H. He and E. A. Garcia. Learning from imbalanced data. TKDE, 21(9):1263-1284, 2009.

[12] K. He, X. Zhang, S. Ren, and J. Sun. Deep residual learning for image ${ }_{435}^{434}$ recognition. In $C V P R, 2016$.

[13] S. Hochreiter and J. Schmidhuber. Long short-term memory. Neurat ${ }^{436}$ Computation, 9(8):1735-1780, 1997.
[14] G. Huang, Z. Liu, L. van der Maaten, and K. Q. Weinberger. Densely connected convolutional networks. In CVPR, 2017.

[15] J. Huo, Y. Gao, Y. Shi, and H. Yin. Cross-modal metric learning for AUC optimization. TNNLS, 29(10):4844-4856, 2017.

[16] S. Ioffe and C. Szegedy. Batch normalization: accelerating deep network training by reducing internal covariate shift. In ICML, 2015.

[17] P. Jeatrakul, K. W. Wong, and C. C. Fung. Classification of imbalanced data by combining the complementary neural network and SMOTE algorithm. In ICONIP, 2010.

[18] S. Jun, B. Park, J. B. Seo, S. Lee, and N. Kim. Development of a computer-aided differential diagnosis system to distinguish between usual interstitial pneumonia and non-specific interstitial pneumonia using texture- and shape-based hierarchical classifiers on HRCT images. Journal of Digital Imaging, 31(2):235-244, 2017.

[19] D. S. Kermany, M. Goldbaum, W. Cai, C. C. Valentim, H. Liang, S. L. Baxter, A. McKeown, G. Yang, X. Wu, F. Yan, J. Dong, M. K. Prasadha, J. Pei, M. Y. Ting, J. Zhu, C. Li, S. Hewett, J. Dong, and K. Zhang. Identifying medical diagnoses and treatable diseases by image-based deep learning. Cell, 172(5):1122-1131, 2018.

[20] S. H. Khan, M. Hayat, M. Bennamoun, F. A. Sohel, and R. Togneri. Costsensitive learning of deep feature representations from imbalanced data. TNNLS, 29(8):3573-3587, 2018

[21] A. Krizhevsky, I. Sutskever, and G. E. Hinton. ImageNet classification with deep convolutional neural networks. In NeurIPS, 2012.

[22] G. Litjens, T. Kooi, B. E. Bejnordi, A. A. A. Setio, F. Ciompi, M. Ghafoorian, J. A. van der Laak, B. van Ginneken, and C. I. Sánchez. A survey on deep learning in medical image analysis. Medical Image Analysis, 42:60-88, 2017

[23] C. Mao, Y. Pan, Z. Zeng, L. Yao, and Y. Luo. Deep generative classifiers for thoracic disease diagnosis with chest $\mathrm{x}$-ray images. arXiv: 1809.07436, 2018.

[24] M. Mazurowski, M. Buda, A. Saha, and M. R. Bashir. Deep learning in radiology: an overview of the concepts and a survey of the state of the art with focus on MRI. Journal of Magnetic Resonance Imaging, 49(4):939954, 2019.

[25] L. F. S. Merchante, Y. Grandvalet, and G. Govaert. An efficient approach to sparse linear discriminant analysis. In ICML, 2012.

[26] D. S. Morillo, A. León-Jiménez, and S. A. Moreno. Computer-aided diagnosis of pneumonia in patients with chronic obstructive pulmonary disease. Journal of the American Medical Information Associations, 20(e1):e111-7, 2013.

[27] E. Naydenova, A. Tsanas, C. Casals-Pascual, and M. D. Vos. Smart diagnostic algorithms for automated detection of childhood pneumonia in resrouce-constrained settings. In IEEE Global Humanitarian Technology Conference (GHTC), 2015.

[28] E. Naydenova, A. Tsanas, S. Howie, C. Casals-Pascual, and M. D. Vos. The power of data mining in diagnosis of childhood pneumonia. Journal of the Royal Society Interface, 13(120):20160266, 2016.

[29] J. O'Connell, K. J. Murphy, S. M. Robinson, K. Iniewski, and M. Bazalova-Carter. Unsupervised learning methods in x-ray spectral imaging meterial segmentation. arXiv: 1904.03701, 2019.

[30] N. R. S. Parveen and M. M. Sathik. Detection of pneumonia in chest Xray images. Journal of X-Ray Science and Technology, 19(4):423-428, 2011.

[31] S. Pouyanfar, S. Sadiq, Y. Yan, H. Tian, Y. Tao, M. P. Reyes, M.-L. Shyu, S.-C. Chen, and S. S. Iyengar. A survey on deep learning: algorithms, techniques, and applications. ACM Computing Surveys, 51(5):Article No. 92, 2019.

[32] P. Rajpurkar, J. Irvin, K. Zhu, B. Yang, H. Mehta, T. Duan, D. Ding, A. Bagul, C. Langlotz, K. Shpanskaya, M. P. Lungren, and A. Y. Ng. CheXNet: radiologist-level pneumonia detection on chest $\mathrm{x}$-rays with deep learning. arXiv: 1711.05225, 2017.

[33] M. Ren, W. Zeng, B. Yang, and R. Urtasun. Learning to reweight examples for robust deep learning. In ICML, 2018.

[34] F. Santosa and W. W. Symes. Linear inversion of band-limited reflection seismograms. SIAM Journal of Scientific and Statistical Computating, 7(4):1307-1330, 1986.

[35] A. A. Saraiva, N. M. F. Ferreira, L. L. de Sousa, N. J. C. Costa, J. V. M. Sousa, D. B. S. Santos, A. Valente, and S. Soares. Classification of images of childhood pneumonia using convolutional neural networks. In International Conference on Bioimaging, 2019. 
[36] S. Shang, L. Chen, K. Zheng, C. S. Jensen, Z. Wei, and P. Kalnis. Parallel trajectory-to-location join. TKDE, 31(6):1194-1207, 2019.

[37] J. Shiraishi, S. Katsuragawa, J. Ikezoe, T. Matsumoto, T. Kobayashi, K. ichi Komatsu, M. Matsui, H. Fujita, Y. Kodera, and K. Doi. Development of a digital image database for chest radiographs with and without a lung nodule: Receiver operating characteristics analysis of radiologists' detection of pulmonary nodules. American Journal of Roentgenology, 174(1):71-74, 2000.

[38] K. Simonyan and A. Zisserman. Very deep convolutional networks for large-scale image recognitions. In $I C L R, 2015$.

[39] R. T. Sousa, O. Marques, F. A. A. Soares, I. I. Sene Jr., L. L. de Oliveira, ${ }^{494}$ and E. S. Spoto. Comparative performance analysis of machine learning ${ }^{495}$ classifiers in detection of childhood pneumonia using chest radiographs.496 Procedia Computer Science, 18:2579-2582, 2013.

[40] C. Szegedy, W. Liu, Y. Jia, P. Sermanet, S. Reed, D. Anguelov, D. Erhan, V. Vanhoucke, and A. Rabinovich. Going deeper with convolutions. In ${ }^{498}$ CVPR, 2015

[41] X. Wang, Y. Peng, L. Lu, Z. Lu, M. Bagheri, and R. M. Summers. ChestX-ray8: Hospital-scale chest $\mathrm{x}$-ray database and benchmarks on weakly-supervised classification and localization of common thorax diseases. In CVPR, 2018.

[42] X. Wang, Y. Peng, L. Lu, Z. Lu, and R. M. Summers. TieNet: textimage embedding network for common thorax disease classification and reporting in chest x-rays. In CVPR, 2018.

[43] World Health Organization. Revised WHO classification and treatment of childhood pneumonia at health facilities: Evidence summaries. http: //www.who.int/iris/handle/10665/137319, 2014.

44] L. Yao, E. Poblenz, D. Dagunts, B. Covington, D. Bernard, and K. Lyman. ${ }_{501}$ Learning to diagnose from scratch by exploiting dependencies among la- ${ }_{502}$ bels. arXiv: 1710.10501, 2017.

[45] X. Yue, M. Xi, B. Chen, M. Gao, Y. He, and J. Xu. A revocable group ${ }^{503}$ signatures scheme to provide privacy-preserving authentications. Mobile504 Networks and Applications, online first, 2019.

[46] J. R. Zech, M. A. Badgeley, M. Liu, A. B. Costa, J. J. Titano, and E. K. Oermann. Variable generalization performance of a deep learning model to detect pneumonia in chest radiographs: a cross-sectional study. PLoS Medicine, 15(11):e1002683, 2018.

[47] T. Zhai, Y. Gao, H. Wang, and L. Cao. Classification of high-dimensional evolving data streams via a resource-efficient online ensemble. DMKD, 31:1242-1265, 2017.

[48] W. Zheng, X. Zhu, G. Wen, Y. Zhu, H. Yu, and J. Gan. Unsupervised feature selection by self-paced learning regularization. Pattern Recognition Letters, online first, 2018.

[49] X. Zhu, X. Li, S. Zhang, Z. Xu, L. Yu, and C. Wang. Graph PCA hashing 506 for similarity search. IEEE Transactions on Multimedia, 19(9):2033- ${ }^{507}$ 2044, 2017.

[50] X. Zhu, S. Zhang, W. He, R. Hu, C. Lei, and P. Zhu. One-step multi-view509 spectral clustering. TKDE, online first, 2018.

[51] X. Zhu, S. Zhang, Y. Li, J. Zhang, L. Yang, and Y. Fang. Low-rank sparse subspace for spectral clustering. TKDE, online first, 2018.

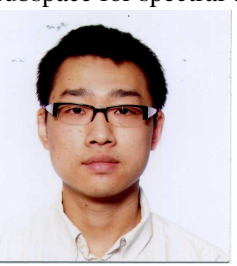

Dr. Hao Wang received his Ph.D. degree in Computer Science from The University of Hong Kong in 2014. He is a Research Scientist at the Inception Institute of Artificial Intelligence. His research interests in- ${ }_{513}$ clude machine learning, and spatio-temporal databases. Email: $:_{514}$ hao.wang@inceptioniai.org.

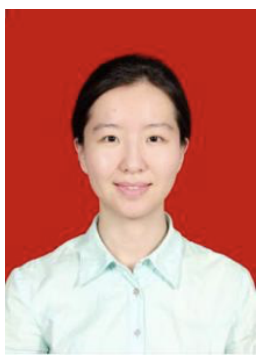

Dr. Yuan-Yuan Yang is with the Department of General Surgery, Fujian Medical University Union Hospital, Fuzhou 350001, P.R. China. Her research interests include health information management, basic and clinical research of pancreatic diseases, and hernia and surgical critical illness. Email: yuuuuan@163.com.

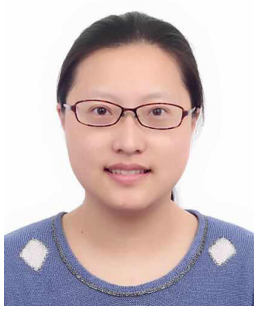

Dr. Yang Pan received her M.D. \& Ph.D. degree in Neurology from Nanjing Medical University in 2016. She is an Associate Chief Physician in the Department of Geriatrics in Nanjing Brain Hospital. Her research interests include Parkinson's disease and medical artificial intelligence. Email: neuro_panyang@163.com.

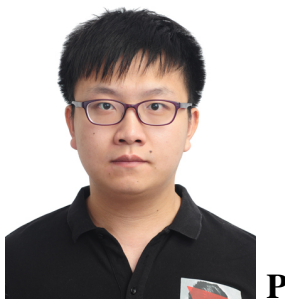

Peng Han received his M.Sc. degree in Computer Science from the Renmin University of China in 2017. Currently, he is pursuing a Ph.D. degree in Computer Science from King Abdullah University of Science and Technology. His research interests include computer vision, recommendation system and distributed deep learning. Email: peng.han@kaust.edu.sa.

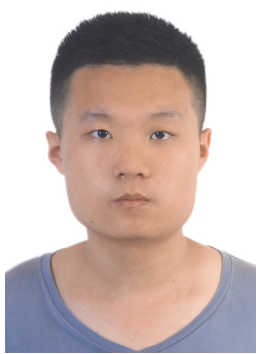

Zhong-Xiao Li received his B.S. degree in bioinformatics at Southern University of Science and Technology (SUSTech) in 2018. He is currently pursuing an M.Sc. degree in computer science at King Abudullah University of Science and Technology (KAUST). His main research interests are machine learning, data mining and bioinformatics. Email: zhongxiao.li@kaust.edu.sa. 


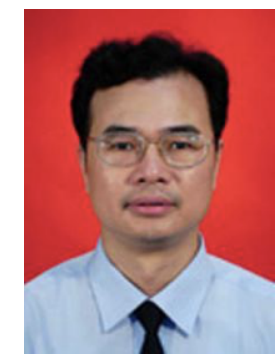
Hospital, Fuzhou 350001, P.R. China. His research interests include health information management, basic and clinical research of pancreatic diseases, and hernia and surgical critical illness. Email: hhuang2@aliyun.com.

Prof. Shun-Zhi Zhu is with the School 527 of Computer and Information Engineering, Xiamen University of Technology. His research interests include data mining, information recommendation, 3D printing, and system engineering. Email: zhusz99@qq.com. 